\title{
Resistive destabilization of cycloidal electron flow and universality of (near-) Brillouin flow in a crossed-field gap
}

\author{
Peggy J. Christenson, ${ }^{\text {a) }}$ David P. Chernin, ${ }^{\text {b) }}$ Allen L. Garner, and Y. Y. Lau ${ }^{\mathrm{c})}$ \\ Department of Nuclear Engineering and Radiological Sciences, University of Michigan, Ann Arbor, \\ Michigan 48109-2104
}

(Received 11 July 1996; accepted 26 August 1996)

\begin{abstract}
It is shown that a small amount of dissipation, caused by current flow in a lossy external circuit, can produce a disruption of steady-state cycloidal electron flow in a crossed-field gap, leading to the establishment of a turbulent steady state that is close to, but not exactly, Brillouin flow. This disruption, which has nothing to do with a diocotron or cyclotron instability, is fundamentally caused by the failure of a subset of the emitted electrons to return to the cathode surface as a result of resistive dissipation. This mechanism was revealed in particle simulations, and was confirmed by an analytic theory. These near-Brillouin states differ in several interesting respects from classic Brillouin flow, the most important of which is the presence of a microsheath and a time-varying potential minimum very close to the cathode surface. They are essentially identical to that produced when (i) injected current exceeds a certain critical value [P. J. Christenson and Y. Y. Lau, Phys. Plasmas 1, 3725 (1994)] or (ii) a small rf electric field is applied to the gap [P. J. Christenson and Y. Y. Lau, Phys. Rev. Lett. 76, 3324 (1996)]. It is speculated that such near-Brillouin states are generic in vacuum crossed-field devices, due to the ease with which the cycloidal equilibrium can be disrupted. Another novel aspect of this paper is the introduction of transformations by which the nonlinear, coupled partial differential equations in the Eulerian description (equation of motion, continuity equation, Poisson equation, and the circuit equation) are reduced to an equivalent system of very simple linear ordinary differential equations. (c) 1996 American Institute of Physics. [S1070-664X(96)01012-9]
\end{abstract}

\section{INTRODUCTION}

When electrons are emitted from a cathode into a vacuum gap to which a dc voltage is applied, and which is immersed in a transverse magnetic field, $B>B_{\mathrm{H}}$, a steady state may be established in which each electron executes a simple, cycloidal orbit and returns to the cathode with the same energy with which it was emitted. Here, $B_{\mathrm{H}}$ is the Hull cutoff magnetic field. ${ }^{1}$ An example is illustrated in Fig. 1. These steady flows are characterized by smoothly varying, time-independent density and potential profiles. Electron density is largest at the cathode and at the turning point at the top of the layer. A steady state of this form can be established if and only if the emitted current density, $J$, is below a critical value, $J_{c}$, that depends on the gap voltage, gap spacing, and magnetic field. ${ }^{2}$ A good estimate of the critical emitted current, for zero beam injection velocity, is obtained from the condition of a zero electric field at the cathode. ${ }^{2-5}$

When the emitted current is above the critical value, the character of the resulting turbulent state, found by computer simulation, is much different. In this case, as shown in Ref. 2 , the electron density builds to the point that a potential minimum is established close to the cathode surface. Those particles out beyond the potential minimum execute orbits that are nearly laminar, with very little energy in the trans-

\footnotetext{
${ }^{a)}$ Present address: Department of Electrical Engineering and Computer Sciences, University of California, Berkeley, California 94720-1770.

${ }^{b}$ Also at Science Applications International Corporation, McLean, Virginia 22102.

c)Also at Applied Physics Program, University of Michigan, Ann Arbor, Michigan 48109.
}

verse direction. Newly emitted particles are (mostly) reflected from the potential minimum, and returned to the cathode. Not all emitted particles are reflected, however. The depth of the potential minimum varies in time, periodically allowing some newly injected particles to pass into the main flow. ${ }^{2,4}$ Of course, some members of the main flow must then return to the cathode to maintain a balance, on average, between the injected and ejected particle fluxes.

The average density of the layer in this state is very close to the classical Brillouin value, given by setting the (average) plasma frequency $\omega_{p}$ equal to the cyclotron frequency $\Omega$. Densities and potentials are not time independent, but rather oscillate with small amplitudes about mean values. ${ }^{4}$ This flow is therefore similar, but not identical, to classical Brillouin flow. ${ }^{6}$ For want of a better term, we shall call it near-Brillouin (nB) flow. [The classical Brillouin flow is characterized by constant density with $\omega_{p}^{2}=\Omega^{2}$ between $x=0$ and $x=D\left[1-\sqrt{1-\left(B_{\mathrm{H}} / B\right)^{2}}\right]$, and by laminar flow velocity $\vec{v}=\hat{y} \Omega x$ in the $E \times B$ direction.]

Additional analytical and numerical studies show that $\mathrm{nB}$ flow, far from being an unusual state, is actually difficult to avoid in practice, even when currents are well below the critical value. For example, it has recently been discovered that the steady-state cycloidal flow below critical current is very unstable, being easily disrupted by the application of a small rf potential. ${ }^{7}$ This disruption is fundamentally one dimensional in nature and therefore has nothing to do with a diocotron or cyclotron instability. ${ }^{8}$ It is caused by the failure of a subset of the emitted electrons to return to the cathode surface due to the action of the rf field. As a result, charge accumulates and the density of the layer builds in time (and 


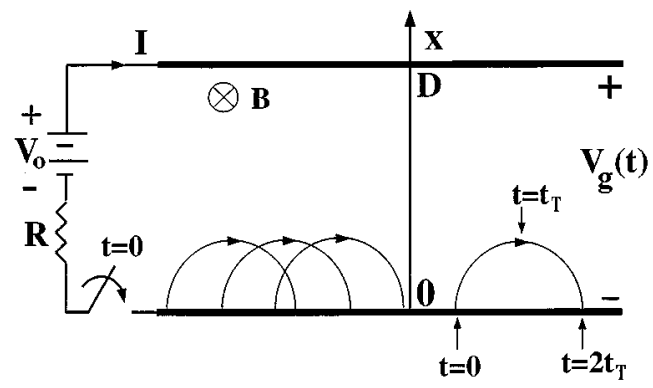

FIG. 1. The model. In the absence of the resistor $R$, an electron emitted at $x=0$ at $t=0$ reaches a maximum height at $t=t_{T}$, and returns to $x=0$ at $t=2 t_{T}$.

smooths in space) to a value near the classical Brillouin limit, with $\omega_{p}^{2}=\Omega^{2}$. It is found by computer simulation that the flow in this case evolves to $\mathrm{nB}$ flow, just as if the injected current were above the critical value.

In the present paper, another way that the cycloidal flow may be disrupted is described. Again, the effect is one dimensional. Specifically, we show that the interaction of an injected beam with an external circuit containing some loss disrupts the cycloidal equilibrium, which, again, develops into $\mathrm{nB}$ flow.

Since the amount of resistance needed to cause this disruption is very small, and since all physical circuits have some dissipation, we conjecture that $\mathrm{nB}$ flow is generic to all vacuum crossed-field devices. In fact, we further conjecture that dissipation, per se, is not required. This is because the energy lost by an electron could instead just as well be stored temporarily in a reactive element. It seems that almost any perturbation encountered in reality on cycloidal flow may drive the flow unstable, resulting in a final state of $\mathrm{nB}$ flow.

This conjectured "universality" of nB flow, if correct, would require, for example, that the electron density near a thermionic cathode in a crossed-field device would depend strongly only on the magnetic field value via the relation $\omega_{p}^{2} \approx \Omega^{2}$ and be insensitive to the cathode temperature, which determines the emission rate by the Richardson-Dushman law. ${ }^{9}$

Note that the fundamental mechanism leading to $\mathrm{nB}$ flow is the same in all cases, namely some or all electrons entering the gap lose energy during their orbit, preventing them from returning to the cathode. Altogether, then, three regimes in which $\mathrm{nB}$ flow is produced have been identified, viz. (i) $J>J_{c},{ }^{2}$ (ii) $J<J_{c}$, with a small rf voltage applied, ${ }^{7}$ and (iii) $J<J_{c}$, with a small resistance in external circuit. In all three cases, the form of $\mathrm{nB}$ flow produced is essentially the same.

In this paper, we concentrate only on the resistive destabilization of the cycloidal flow. We shall first show, via computer simulation using the one-dimensional code PDP $1,{ }^{10}$ that a very small resistance $R$ may destabilize the cycloidal flow. Examination of the computer runs reveals that the collapse of the cycloidal flow occurs concomitantly when at least some of the emitted electrons fail to return to the cathode. In the case of low injected current, it is the leading part of the electron flow (i.e., the first emitted electrons) that fails to return to the cathode, whereas in the case of high injected current, it is the trailing part of the electron flow (i.e., the electrons emitted subsequently) that fails to return to the cathode. (By high injection current, we mean a significant fraction of the critical current, as defined more precisely below.) Given an injection current, there is a threshold resistance beyond which the cycloidal flow collapses into the $\mathrm{nB}$ flow. We next develop an analytic theory to calculate this threshold resistance. It turns out that, up to the time $\left(t_{T}\right)$ that the lead electron takes to reach the maximum excursion from the cathode (Fig. 1), the formulation can be done exactly in terms of very simple, linear ordinary differential equations. Space charge effects are fully accounted for and no linearization is performed. In effect, we have transformed the nonlinear, coupled partial differential equations in the Eulerian description (equation of motion, continuity equation, Poisson equation, and the circuit equation) into an equivalent system of linear ordinary differential equations before orbit crossing occurs. For $t>t_{T}$, the formulation is approximate. These linear equations are then solved, both numerically and perturbatively, to yield the threshold resistance by requiring that the lead electrons not to return to the cathode in the case of low injection current. These analytic results were found to be in good agreement with particle simulation results for moderately low values of $J$ up to about $1 / 3 J_{c}$.

In Sec. II, we present the model and the simulation results. In Sec. III, we formulate the analytic theory and present the solution, both by direct numerical integration and by asymptotic expansion. Concluding remarks are given in Sec. IV.

\section{MODEL AND SIMULATION RESULTS}

The model used in the simulations is shown in Fig. 1. It is a simple one-dimensional anode-cathode gap of separation $D=0.00216 \mathrm{~m}$ and electrode area $A=0.003122 \mathrm{~m}^{2}$, that is immersed in a constant transverse insulating magnetic field $B=0.27 \mathrm{~T}\left(B>B_{\mathrm{H}}\right)$. An external circuit consisting of a constant resistance $R$ and a steady voltage $V_{0}=12000 \mathrm{~V}$ is connected to the gap. This gap voltage gives the Hull cutoff magnetic field $B_{\mathrm{H}}=\left(2 m V_{0} / e D^{2}\right)^{1 / 2}=0.171 \mathrm{~T}$. At time $t=0$, the switch is closed (Fig. 1). Electrons begin to be injected into the gap at a constant rate with a constant current density $J$, and the gap voltage, $V_{g}(t)$, begins to ramp from zero to its equilibrium value as current flows through the external circuit. The cathode potential is always held at zero volts and the anode potential is allowed to fluctuate. It must be remembered that due to the magnetic field that insulates the gap against electron flow, the current, $I$, flowing through the external circuit (and across the gap) is the displacement current induced at the anode. This displacement current has two components, the familiar term $C d V_{g} / d t$ and the induced current as a result of electron motion in the gap. Here, $C=A \epsilon_{0} / D$ is the capacitance in vacuum. One further simplification of our model is that the electrons are all emitted at a single velocity $u_{0}$. The electron emission energy is $0.5 \mathrm{eV}$, which implies a critical current density, $J_{c}=229800 \mathrm{~A} / \mathrm{m}^{2}$, or critical current $I_{c}=A J_{c}=717 \mathrm{~A} .{ }^{2,4}$ We restrict our studies to $J<J_{c}$.

The computer simulations were done using the onedimensional electrostatic particle-in-cell (PIC) code, PDP1. ${ }^{10}$ 

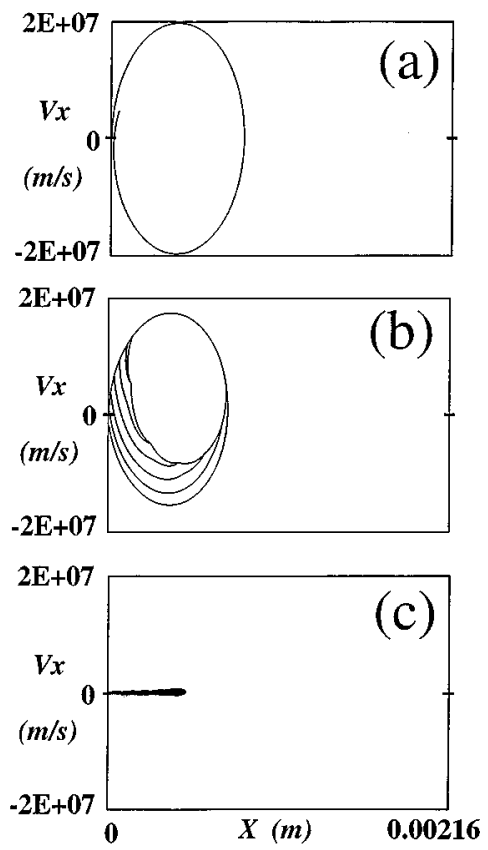

FIG. 2. Phase space plots (a) immediately after one cycloidal orbit, (b) after ten cycloidal orbits, and (c) in the final turbulent, near-Brillouin state.

The remainder of this section is a discussion of the observations from the PIC simulation. In all cases we studied, at a fixed value of the injection current density $J$, we found that there is a threshold resistance above which the electron flow becomes turbulent (Fig. 2) and below which the cycloidal electron flow remain stable and time independent (Fig. 3). In all cases where the cycloidal electron flow becomes turbulent, there is buildup of space charge within the gap that is caused by the failure in some portion of the emitted electrons to return to the cathode. The final turbulent state is always the $\mathrm{nB}$ state.

The route to the $\mathrm{nB}$ state differs in detail, depending on the level of injected current. For example, at the intermediate level of injection current (roughly in the range $0.05 J_{c}<J$ $<0.7 J_{c}$, in which most of the simulations were performed), the failure of the lead electron, defined as that emitted from the cathode at $t=0$, to return to the cathode signals the sub-

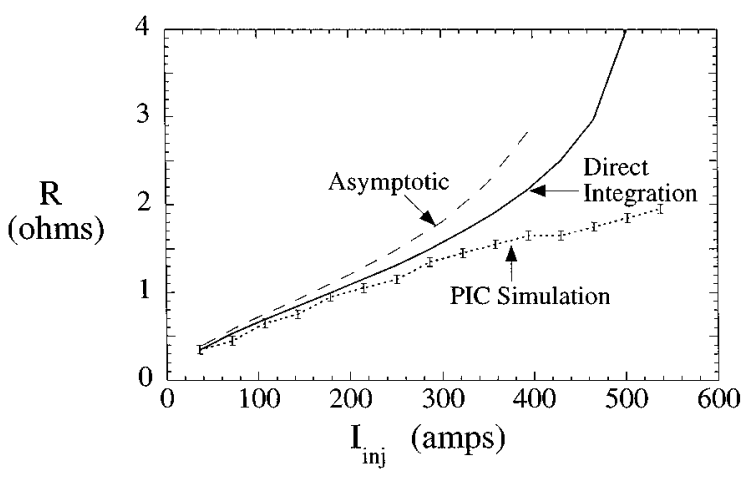

FIG. 3. Threshold resistance for the onset of turbulence according to particle simulation. Also shown is the threshold resistance to prevent the lead electron from returning to the cathode, according to the analytic theory. sequent development into the turbulent $\mathrm{nB}$ state. Figure 2 shows the evolution of the phase-space plots, $v_{x}$ vs $x$, of the ensemble of electrons. Figure 2(a) is taken at $t=0.15 \mathrm{ns,} \mathrm{Fig.}$ 2(b) at $t=1.5 \mathrm{~ns}$, and finally Fig. 2(c) at $t=20 \mathrm{~ns}$. The case shown in these figures is for an emitted current of $J=15 \%$ of $J_{c}$ with an external resistance of $1 \Omega$. This set of plots is typical of the onset of the instability in the range of moderately low emitted electron current. Figure 2(a) shows the lead electrons failing to return to the cathode. In Fig. 2(b) we see that after many cycles, there is a buildup of space charge within the gap as subsequent electrons fail to return to the cathode. And, finally, in Fig. 2(c) we see the phase-space plot that is typical of all $\mathrm{nB}$ flows.

Since this $\mathrm{nB}$ flow is essentially identical to that produced when (i) injected current $J$ exceeds a certain critical value $J_{c}{ }^{2}$ or (ii) a small rf electric field is applied to the gap, ${ }^{7}$ we recapitulate the main properties of the $\mathrm{nB}$ flow that were observed from the simulation: ${ }^{4}$ (a) The electrons in the gap display time-dependent, turbulent behavior. (b) The particle phase space is greatly contracted in $v_{x}$, and the electron density profile flattens out in $x$, extending from the cathode to roughly the classical electron sheath thickness, $D\left[1-\sqrt{1-\left(B_{\mathrm{H}} / B\right)^{2}}\right]$. The latter quantity is less than the maximum excursion, from the cathode, of the electron orbit in the stable, time-independent case. (c) The time-averaged plasma frequency satisfies $\omega_{p}=\Omega$, the cyclotron frequency. (d) The direction of the cathode surface electric field, and the sign of the cathode surface charge density, indicate the presence of a potential minimum in the immediate vicinity of the cathode surface. Thus, a microsheath is always present. (e) The potential minimum in the microsheath is oscillatory, and its depth is on the order of the injection energy of the electrons. (f) The space charge $Q_{G}$ in the gap, the surface charge on the cathode $Q_{K}$, and the total charge associated with the gap, $Q_{T}=Q_{K}+Q_{G}$, are all nearly constant. Moreover, $Q_{T}$ is approximately equal to $C V_{0}, C$ being the vacuum capacitance.

Returning to the resistive destabilization of the cycloidal flow, we show in Fig. 3 the threshold resistance for the onset of turbulence obtained from the PIC simulation. Also shown are the results obtained from an analytic theory, which gives the threshold resistance required to prohibit the lead electron from returning to the cathode in the intermediate current range. The small numerical values of the threshold resistor, of order only a few ohms as shown in Fig. 3, prompted our speculation that the $\mathrm{nB}$ state is almost inevitable in practice.

At high injection currents, roughly in the range $0.7 J_{c}<J<J_{c}$, the leading electrons always return to the cathode at the critical resistance; although, with a large enough external resistance it is possible to cause the lead electrons to fail to return to the cathode. For the lowest external resistance that causes instability here, it is a group of electrons released after time $t=0$ that fail to return to the cathode and cause a buildup of space charge within the gap, thereby triggering the disruption of the cycloidal flow.

Finally, for very low injection currents, for $J \approx 0$ up to about $0.05 J_{c}$, the cycloidal flows are still unstable to an external resistance; however, the criterion is no longer simply that the leading electrons do not return to the cathode. 
With a small resistance, while the electrons emitted at time $t=0$ may not return to the cathode after one Larmor cycle (or at any time thereafter), the perturbation in space charge is not sufficient to trigger a collapse of the cycloidal flow. For the cycloidal flow to evolve to the $\mathrm{nB}$ state, the external resistance must be large enough that the energy dissipation in the resistor by the very small displacement current is sufficient to cause electrons that are emitted after one Larmor period to fail to return to the cathode in addition to the lead electron. Thus as the emitted electron density goes to zero, the critical resistance must increase.

We should point out that extensive numerical tests (e.g., by varying particle weighting, or time step, up to two orders of magnitude, or cell size, or combination of such) have been performed ${ }^{4}$ to show that the $\mathrm{nB}$ states and the mechanisms that lead to them are not a numerical artifact.

In the next section, we present the analytic theory that determines the threshold resistance above which the lead electron does not return to the cathode, and the results of this theory are in good agreement with simulation in the intermediate injection current regime (Fig. 3).

\section{ANALYTIC THEORY}

In most of our simulations, strong hints of instability emerge once the first electrons fail to return to the cathode at the end of their first cycloidal period [Fig. 2(a)]. This would allow progressive buildup of the space charge in the gap [Fig. 2(b)] and trigger breakdown of the cycloidal flow [Fig. 2(c)]. Given below is an analytic theory that describes the onset of the resistive instability according to this scenario. When such a theory is used to deduce the threshold resistance for the onset of the instability, good agreement with simulation is obtained.

\section{A. Formulation}

We shall use the Lagrangian description to formulate the circuit equation, the force law (with the continuity equation taken into account), and the Poisson equation. Since we are dealing with a one-dimensional model with a monoenergetic emission velocity, an electron that is released at $x=0$ at time $t=t_{i}$ represents the entire electron sheet that is released from $x=0$ at $t=t_{i}$. Let $x_{i}\left(t, t_{i}\right)$ be the $x$ coordinate of this electron sheet at time $t\left(t>t_{i}\right)$.

Consider the electrons that are released from $x=0$ (Fig. 1) between the time interval $t_{i}$ and $t_{i}+\Delta t_{i}$. The thickness of this incremental electron sheet is $\Delta x_{i}=u_{0} \Delta t_{i}$ and the surface charge density (in $\mathrm{C} / \mathrm{m}^{2}$ ) of this incremental electron sheet is

$$
\sigma_{i}=\rho_{0} \Delta x_{i}=\rho_{0} u_{0} \Delta t_{i}=J \Delta t_{i},
$$

where $u_{0}$ and $\rho_{0}$ is, respectively, the initial velocity and the initial volume charge density (in $\mathrm{C} / \mathrm{m}^{3}$ ), and $J=\rho_{0} u_{0}$ is the injection current density that is constant in space and in time $(t>0)$. By convention, we take $\sigma, \rho, J$, and the electronic charge, $e$, to be positive. Charge conservation (i.e., the continuity equation) is automatically satisfied if the same $\sigma_{i}$ is used when this incremental electron sheet moves within the gap. Motion of this electron sheet provides an induced current, given by

$$
I_{i}(\text { induced })=\left(\frac{A \sigma_{i}}{D}\right) \frac{\partial x_{i}\left(t, t_{i}\right)}{\partial t},
$$

by Ramo's theorem. ${ }^{11,12}$ The total induced current may be obtained by summing all such charge sheets within the gap:

$$
\begin{aligned}
I \text { (induced) } & =\sum_{i}\left(\frac{A \sigma_{i}}{D}\right) \frac{\partial x_{i}\left(t, t_{i}\right)}{\partial t} \\
& =\frac{A J}{D} \sum_{i}\left(\Delta t_{i}\right) \frac{\partial x_{i}\left(t, t_{i}\right)}{\partial t},
\end{aligned}
$$

where we have used Eq. (1).

The current $I(t)$ that passes through the resistor (Fig. 1) is the sum of Eq. (3) and the familiar term $C d V_{g} / d t$, where $C=A \epsilon_{0} / D$ is the gap capacitance. ${ }^{11,12}$ Thus, we have

$$
I(t)=C \frac{d V_{g}(t)}{d t}+\frac{A J}{D} \int_{0}^{t} d t_{0} \frac{\partial x\left(t, t_{0}\right)}{\partial t},
$$

where we have taken the continuum limit by replacing the summation in Eq. (3) with the integral in Eq. (4). In Eq. (4), $x\left(t, t_{0}\right)$ is the $x$ coordinate of an electron at time $t$ that was emitted from $x=0$ at an earlier time $t_{0}$. From this definition, it is clear that

$$
x\left(t_{0}, t_{0}\right)=0,\left.\quad \frac{\partial x\left(t, t_{0}\right)}{\partial t}\right|_{t=t_{0}}=u_{0} .
$$

The Kirchhoff voltage law, $V_{0}=V_{g}+R I$ (cf. Fig. 1) then reads as

$$
R C \frac{d V_{g}(t)}{d t}+V_{g}(t)=V_{0}-R \frac{A J}{D} \int_{0}^{t} d t_{0} \frac{\partial x\left(t, t_{0}\right)}{\partial t},
$$

where we have used Eq. (4). Equation (6) is the circuit equation that governs the evolution of the gap voltage.

The equation of motion for a single electron reads as

$$
\frac{\partial^{2} x_{i}\left(t, t_{i}\right)}{\partial t^{2}}+\Omega^{2} x_{i}\left(t, t_{i}\right)=-\frac{e}{m} E\left(x_{i}, t\right),
$$

where $\Omega=e B / m$ is the electron cyclotron frequency and $E$ is the total electric field, including the time-varying space charge field that is acting on that electron. Implicit in Eq. (7) is the neglect of the self-magnetic field and relativistic effects. The total electric field $E$ at position $x=x_{i}$ is the sum of the vacuum field $-V_{g} / D$ and the space charge field. It is derived in Appendix A [cf. Eq. (A6) for $t<t_{T}$, where $t_{T}$ is the time it takes the lead electron to reach the maximum excursion. See Fig. 1]. In the continuum limit, Eq. (7) then reads as

$$
\begin{aligned}
\frac{\partial^{2} x\left(t, t_{0}\right)}{\partial t^{2}}+\Omega^{2} x\left(t, t_{0}\right)= & \frac{e}{m}\left(\frac{V_{g}(t)}{D}+\frac{J}{\epsilon_{0} D} \int_{0}^{t} d t_{0} x\left(t, t_{0}\right)\right. \\
& \left.-\frac{J t_{0}}{\epsilon_{0}}\right), \quad 0 \leqslant t_{0} \leqslant t \leqslant t_{T}
\end{aligned}
$$

Note that Eqs. (6) and (8) are the governing integrodifferential equations for $V_{g}(t)$ and $x\left(t, t_{0}\right)$. They are linear, but they are also exact, as no linearization has been made and the time-varying space charge field is accounted for completely. 
A simple transformation, to be introduced shortly, cast these two equations into linear ordinary differential equations.

To simplify the notation, we shall use the dimensionless quantities defined as follows:

$$
\begin{aligned}
& \tau \equiv \Omega t, \quad \tau_{0} \equiv \Omega t_{0}, \quad \tau_{T} \equiv \Omega t_{T}, \quad \xi\left(\tau, \tau_{0}\right) \equiv x\left(t, t_{0}\right) / D, \\
& \widetilde{u_{0}} \equiv \frac{u_{0}}{\Omega D}, \quad \widetilde{V}_{0} \equiv \frac{e V_{0}}{m \Omega^{2} D^{2}}, \quad \widetilde{V}_{g} \equiv \frac{e V_{g}}{m \Omega^{2} D^{2}}, \\
& \widetilde{J} \equiv \frac{e J}{m \epsilon_{0} \Omega^{3} D}, \quad r \equiv R C \Omega .
\end{aligned}
$$

We shall also introduce the function

$$
p(\tau)=\int_{0}^{\tau} d \tau_{0} \xi\left(\tau, \tau_{0}\right),
$$

in terms of which we can nondimensionalize Eq. (8) as

$$
\begin{gathered}
\frac{\partial^{2} \xi\left(\tau, \tau_{0}\right)}{\partial \tau^{2}}+\xi\left(\tau, \tau_{0}\right)=\widetilde{V}_{g}(\tau)+\widetilde{J}\left[p(\tau)-\tau_{0}\right], \\
0<\tau_{0}<\tau<\tau_{T} .
\end{gathered}
$$

It is straightforward to verify from Eq. (10) that

$$
\begin{aligned}
& \frac{d p(\tau)}{d \tau}=\int_{0}^{\tau} d \tau_{0} \frac{\partial \xi\left(\tau, \tau_{0}\right)}{\partial \tau}, \\
& \frac{d^{2} p(\tau)}{d \tau^{2}}={\widetilde{u_{0}}}+\int_{0}^{\tau} d \tau_{0} \frac{\partial^{2} \xi\left(\tau, \tau_{0}\right)}{\partial \tau^{2}},
\end{aligned}
$$

upon using the initial conditions, Eq. (5). In terms of the dimensionless quantities defined in Eq. (9), the circuit equation (6) is normalized to read as

$$
r \frac{d \widetilde{V}_{g}(\tau)}{d \tau}+\widetilde{V}_{g}(\tau)=\widetilde{V}_{0}-r \widetilde{J} \frac{d p(\tau)}{d \tau},
$$

where we have used the first part of Eq. (12). The governing equation for $p(\tau)$ may be obtained by integrating Eq. (11) with respect to $\tau_{0}$ from zero to $\tau$. This yields

$$
\begin{aligned}
\frac{d^{2} p(\tau)}{d \tau^{2}}+p(\tau)= & \widetilde{u}_{0}+\tau \widetilde{V}_{g}(\tau)+\widetilde{J}\left(\tau p(\tau)-\frac{\tau^{2}}{2}\right), \\
& 0<\tau<\tau_{T},
\end{aligned}
$$

where we have used the second part of Eq. (12).

Note that Eqs. (13) and (14) are linear ordinary differential equations governing the evolution of $p(\tau)$ and $\widetilde{V}_{g}(\tau)$. The solution of $p(\tau)$ and $\widetilde{V}_{g}(\tau)$ may then be used in the right-hand member of Eq. (11), which then becomes a very simple second-order ordinary differential equation in $\tau$ with $\tau_{0}$ being treated as a parameter. These equations, (11), (13), and (14), are exact; no approximation has been introduced to derive them. The initial conditions for $\xi\left(\tau, \tau_{0}\right), p(\tau)$, and $\widetilde{V}_{g}(\tau)$ are

$$
\begin{aligned}
& \xi\left(\tau_{0}, \tau_{0}\right)=0,\left.\quad \frac{\partial \xi\left(\tau, \tau_{0}\right)}{\partial \tau}\right|_{\tau=\tau_{0}}=\widetilde{u_{0}}, \\
& p(0)=0,\left.\quad \frac{d p(\tau)}{d \tau}\right|_{\tau=0}=0,
\end{aligned}
$$

$$
\widetilde{V}_{g}(0)=0 .
$$

Condition (15a) follows Eq. (5), condition (15b) follows (10) and (12), and condition (15c) follows (13) whose right-hand member does not exhibit a delta function at $\tau=0$ [and hence $\widetilde{V}_{g}(\tau)$ must be continuous at $\tau=0$, leading to Eq. (15c)].

If we examine only the trajectory of a lead electron, we set $\tau_{0}=0$ in Eq. (11). With $\xi(\tau) \equiv \xi(\tau, 0)$, Eq. (11) gives the following equation for the trajectory of the lead electron:

$$
\frac{d^{2} \xi(\tau)}{d \tau^{2}}+\xi(\tau)=\widetilde{V}_{g}(\tau)+\widetilde{J} p(\tau), \quad 0<\tau<\tau_{T} .
$$

Since the three governing equations, (13), (14), and (16) for $\widetilde{V}_{g}(\tau), p(\tau)$, and $\xi(\tau)$ are exact, we have in effect transformed the nonlinear partial differential equations in the equivalent Eulerian description (Euler's equation of motion, continuity equation, Poisson equation, and the circuit equation, in a finite geometry) to these three simple linear ordinary differential equations for $0<\tau<\tau_{T}$.

The force law for the lead electrons, Eq. (16), may be extended to $\tau>\tau_{T}$ in an approximate manner. Assuming that the electron orbits in the $x-y$ plane are symmetrical about $\tau=\tau_{T}$ (or $t=t_{T}$ in Fig. 1), we find that for $\tau>\tau_{T}$ there is an additional term attached to the right-hand member of Eq. (16). In Appendix A, we show that this additional term is $-2 \widetilde{J}\left(\tau-\tau_{T}\right)$. Thus, Eq. (16) may be extended to read [cf. Eq. (A8)] as

$$
\begin{gathered}
\frac{d^{2} \xi(\tau)}{d \tau^{2}}+\xi(\tau)=\widetilde{V}_{g}(\tau)+\widetilde{J}\left[p(\tau)-2\left(\tau-\tau_{T}\right) h\left(\tau-\tau_{T}\right)\right] \\
0<\tau<2 \tau_{T}
\end{gathered}
$$

where $h$ is the unit step function: $h(x)=0, x<0 ; h(x)=1$, $x>0$.

Similarly, the circuit equation (14) needs to be extended to $\tau>\tau_{T}$. However, for the purpose of calculating the lead electrons' trajectories, this is unnecessary. The underlying reason is that the numerical value of $\widetilde{J}$ is much less than unity, even if the injection current reaches the critical value [see Eq. (19) below]. The modification to Eq. (14) for $\tau>\tau_{T}$ is the introduction of an additional term to its right-hand member, and that term is proportional to $\widetilde{J}$. Such a modification in the solution of $p(\tau)$ will hardly change the solution of $\xi(\tau)$ in Eq. (17) since $p(\tau)$ in Eq. (17) is already multiplied by the small quantity $\widetilde{J}$. Using this argument, we may even simplify Eq. (14) to read as

$$
\frac{d^{2} p(\tau)}{d \tau^{2}}+p(\tau)=\widetilde{u}_{0}+\tau \widetilde{V}_{g}(\tau), \quad 0<\tau \lesssim 2 \tau_{T},
$$

and at the same time extend the interval of validity to $\tau \approx 2 \tau_{T}$, when the lead electrons are about to return to the cathode surface.

We shall solve Eqs. (13), (17), and (18) subject to the initial conditions (15), and determine the threshold resistance $r$ beyond which the lead electrons fail to return to the cathode surface. 


\section{B. Numerical results}

We shall first show the results of a direct numerical integration of Eq. (13), (17), and (18). This allows immediate comparison with the particle simulation results shown in Sec. II. We next develop the perturbative solution to these equations based on the smallness of the dimensionless parameter $\widetilde{J}$. Specifically, we obtain the approximate solution of $\xi(\tau)$ explicitly.

Using the parameters specified in Sec. II, we obtain the following numerical values of the dimensionless quantities defined in Eq. (9),

$$
\begin{aligned}
& \widetilde{J}=2.75 \times 10^{-5} \times\left(\begin{array}{ll}
I / 1 & \mathrm{~A}
\end{array}\right), \quad r=0.608 \times(R / 1 \Omega), \\
& \widetilde{V}_{0}=0.201, \quad \widetilde{u_{0}}=0.0041 \text {. }
\end{aligned}
$$

The critical current is $I_{c}=717 \mathrm{~A}$, and $\widetilde{J}<0.02$ if the injected current $I<I_{c}$. We take $r$ and $\widetilde{V}_{0}$ to be of order unity; $\widetilde{J}$ and $\tilde{u}_{0}$ to be much less than unity.

\section{Direct integration}

We numerically integrate Eqs. (13), (17), and (18) up to the neighborhood of $\tau=2 \tau_{T}$, where $\tau_{T}$ is obtained from the Llewellyn solutions ${ }^{2,12,13}$ for cycloidal flows. We remark that $\tau_{T}$ approaches $\pi$ in the limit of zero injection current. We then determine the value of the resistor $R$ beyond which $\xi(\tau)>0$ for $\tau$ around $2 \tau_{T}$. This is then the threshold resistance that prohibits the lead electron from returning to the cathode according to the analytic formulation. The result is shown in Fig. 3. There we see that the threshold resistance for the moderately low injection current cases is indeed determined by the failure of the lead electron to return to the cathode surface. At high injection current, the lead electron strikes the cathode after the first cycloidal period while the subsequent electrons will miss the cathode according to our simulation results. In such a case, the lead electron's failure to reach the cathode is NOT the criterion in the determination of the threshold resistor. This is the main reason for the discrepancy between the simulation results and the analytic theory shown in Fig. 3 at high injection current levels.

\section{Perturbative solutions}

Since $\widetilde{J}$ is a small parameter according to Eq. (19), we expand the solutions in power series of $\widetilde{J}$ :

$$
\begin{aligned}
& \xi(\tau)=\xi_{0}(\tau)+\widetilde{J} \xi_{1}(\tau)+\widetilde{J}^{2} \xi_{2}(\tau)+\cdots, \\
& p(\tau)=p_{0}(\tau)+\widetilde{J} p_{1}(\tau)+\widetilde{J}^{2} p_{2}(\tau)+\cdots, \\
& \widetilde{V}_{g}(\tau)=\widetilde{V}_{g 0}(\tau)+\widetilde{J} \widetilde{V}_{g 1}(\tau)+\widetilde{J}^{2} \widetilde{V}_{g 2}(\tau)+\cdots .
\end{aligned}
$$

For simplicity, we now assume that the electrons are emitted with zero energy. Thus $\widetilde{u_{0}}=0$ and all of the initial conditions for $\xi, p$, and $\vec{V}_{g}$ become homogeneous [cf. Eq. (15)]. With this assumption, substitution of the expansion (20) into (13), (17), and (18) yields, to the zeroth order in $\widetilde{J}$,

$$
\begin{aligned}
& r \frac{d \widetilde{V}_{g 0}(\tau)}{d \tau}+\widetilde{V}_{g 0}(\tau)=\widetilde{V}_{0}, \\
& \frac{d^{2} \xi_{0}(\tau)}{d \tau^{2}}+\xi_{0}(\tau)=\widetilde{V}_{g 0}(\tau), \quad 0<\tau \lesssim 2 \pi,
\end{aligned}
$$

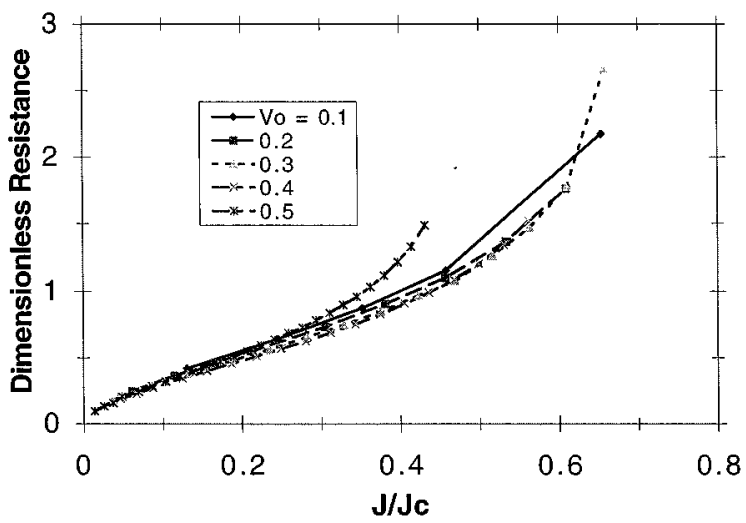

FIG. 4. Threshold resistance, as a function of injection current in units of the critical current at various values of $\widetilde{V}_{0}=\left(B_{\mathrm{H}} / B\right)^{1 / 2} / 2$, to prevent the lead electron from returning to the cathode according to the asymptotic theory.

$$
\frac{d^{2} p_{0}(\tau)}{d \tau^{2}}+p_{0}(\tau)=\tau \widetilde{V}_{g 0}(\tau), \quad 0<\tau \lesssim 2 \pi,
$$

and to the first order in $\widetilde{J}$,

$$
\begin{gathered}
r \frac{d \widetilde{V}_{g 1}(\tau)}{d \tau}+\widetilde{V}_{g 1}(\tau)=-r \frac{d p_{0}(\tau)}{d \tau}, \\
\frac{d^{2} \xi_{1}(\tau)}{d \tau^{2}}+\xi_{1}(\tau)=\widetilde{V}_{g 1}(\tau)+\left[p_{0}(\tau)-2(\tau-\pi) h(\tau-\pi)\right], \\
0<\tau \lesssim 2 \pi, \\
\frac{d^{2} p_{1}(\tau)}{d \tau^{2}}+p_{1}(\tau)=\tau \widetilde{V}_{g 1}(\tau), \quad 0<\tau \lesssim 2 \pi,
\end{gathered}
$$

where we have further approximated $\tau_{T}=\pi$.

The zeroth-order equations (21a)-(21c) are easily solved to yield closed form solutions for $\widetilde{V}_{g 0}(\tau), \xi_{0}(\tau)$, and $p_{0}(\tau)$. Use of $p_{0}(\tau)$ in (22a) allows us to solve for $\bar{V}_{g 1}(\tau)$ in closed form. Substitution of these algebraic expressions of $p_{0}(\tau)$ and $\widetilde{V}_{g 1}(\tau)$ into (22b) enables us to obtain a closed form solution for $\xi_{1}(\tau)$ (which is best obtained by a symbolic manipulation program because of the tedious, though straightforward algebra). Appendix B records the zeroth- and first-order solutions. Thus, from these closed form solutions, we obtain, to first order in $\widetilde{J}$,

$$
\xi(\tau)=\xi_{0}(\tau)+\widetilde{J} \xi_{1}(\tau),
$$

which determines the condition on the threshold resistance, at a given $\widetilde{J}$, by requiring that $\xi(\tau)>0$ for $\tau$ around $2 \pi$. The result is also shown in Fig. 3, which shows good agreement between this asymptotic theory, direct numerical integration, and simulation results for $I$ up to about $240 \mathrm{~A}$, which is $1 / 3 I_{c}$. The discrepancy between the asymptotic results and direct integration shown in Fig. 3 arises from the additional approximations that were introduced in the asymptotic theory: (i) $u_{0}$ is assumed zero, (ii) $\tau_{T}$ is assumed to be $\pi$, and (iii) only zeroth- and first-order terms in $\widetilde{J}$ are kept.

The soundness of the perturbative solution under the assumption $u_{0}=0$ enables us to construct the universal curves (Fig. 4) on the normalized threshold resistance $r$, based on Eq. (23), as a function of $J / J_{c}$ at various values of $\widetilde{V}_{0}$. In Fig. 
4, $\widetilde{V}_{0}$ is restricted to less than $\frac{1}{2}$ since $\widetilde{V}_{0}=\left(B_{\mathrm{H}} / B\right)^{2} / 2$ in the absence of a resistor (Fig. 1). It is interesting to note that the curves in Fig. 4 are insensitive to $\widetilde{V}_{0}$ for $J / J_{c}<0.3$, and these curves are expected to give a reasonable indication of the threshold resistor in this low-current regime.

\section{CONCLUDING REMARKS}

Throughout the years, two types of equilibrium have been envisioned in electron flows in a crossed electric and magnetic field. The first one is the "multistream model," in which the electrons are emitted from the cathode and return to the cathode in the equilibrium state, as studied in this paper. The second one is the "single stream model" or "Brillouin model" in which the electrons are injected externally in the form of a parallel, laminar flow in equilibrium. Extensive numerical simulations, mostly done in the past with the inclusion of two-dimensional effects for either magnetron ${ }^{14}$ or crossed-field amplifier, ${ }^{15}$ did show that the nonlinear states contain a very significant component of Brillouin flow. In these simulations, the electrons are either emitted from the cathode (sole) or injected externally. The present paper, together with the studies reported in Refs. 2, 4 , and 7 , show that the multistream equilibrium is highly unstable with respect to one-dimensional perturbations. Virtually any perturbation encountered in practice would lead to a collapse into the $\mathrm{nB}$ state, which consists of the classical Brillouin flow in the mean, superimposed by a turbulent background. Interestingly, such a collapse appears to be insensitive to the level of the emitted current.

It can be shown that, for the same gap spacing, the same voltage, the same magnetic field, and the same circulating current, the multistream equilibrium has a higher kinetic energy density and higher electrostatic energy density than the classical Brillouin flow. It is, therefore, understandable that all multistream flows have the tendency to relax to the $\mathrm{nB}$ state, whether by dissipation or by rf modulation. In this sense, the $\mathrm{nB}$ state is an "attractor". ${ }^{16}$

\section{ACKNOWLEDGMENTS}

This work was supported by the Naval Research Laboratory/Office of Naval Research, by the Department of Defense Augmentation Awards for Science and Engineering Training, and by the Naval Surface Warfare Center/Crane Division.

\section{APPENDIX A: EVALUATION OF THE ELECTRIC FIELDS}

In this appendix, we evaluate the electric fields in the planar model, for both $t<t_{T}$, and $t_{T}<t<2 t_{T}$ (Fig. 1). We shall first calculate the electric field due to a single electron sheet with surface charge density $-\sigma_{j}$ located at $x=x_{j}$ inside the planar gap of width $D$. A voltage $V_{g}$ is imposed across the gap. Thus, we solve the Poisson equation,

$$
\frac{d^{2} \phi(x)}{d x^{2}}=\frac{\sigma_{j}}{\epsilon_{0}} \delta\left(x-x_{j}\right), \quad \phi(0)=0, \quad \phi(D)=V_{g},
$$

for the electrostatic potential $\phi(x)$, from which we obtain the electric field $E(x)$,

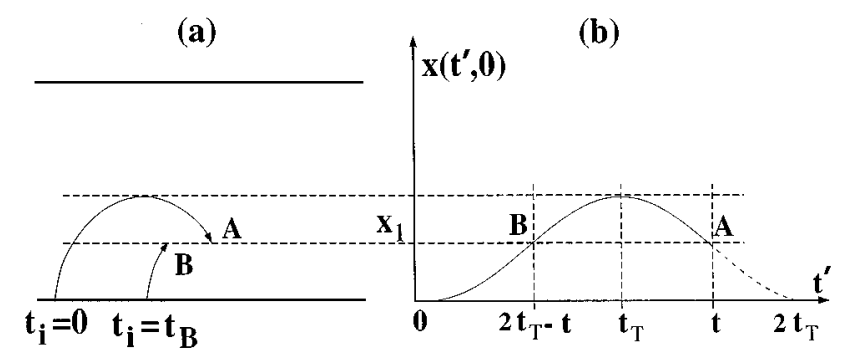

FIG. 5. (a) Electrons $A$ and $B$ arriving at $x=x_{1}$ at the same time $t$. Lead electron $A$ is injected at $t=t_{i}=0$, and electron $B$ is injected at time $t_{B}$ later. (b) Trajectory of lead electron $A$, under the assumption that its orbit is symmetrical about $t=t_{T}$.

$$
\begin{aligned}
& E(x)=-\frac{d \phi(x)}{d x}=-\frac{V_{g}}{D}+E_{j}(x), \\
& E_{j}(x)= \begin{cases}\frac{\sigma_{j}}{\epsilon_{0} D}\left(D-x_{j}\right), & x<x_{j}, \\
-\frac{\sigma_{j}}{\epsilon_{0} D} x_{j}, & x>x_{j},\end{cases}
\end{aligned}
$$

where $\delta$ is the Dirac delta function and $E_{j}$ is the space charge field due to the charge sheet $\sigma_{j}$.

For $t<t_{T}$, the lead electron has not yet reached the maximum excursion $x_{T}$. The electric field $E\left(x_{i}\right)$ at the position $x_{i}$ is due to the electron sheets located both above $x_{i}$ and below $x_{i}$. Upon using Eqs. (A2) and (A3), we then have

$$
E\left(x_{i}\right)=-\frac{V_{g}}{D}+\sum_{x_{i}<x_{j}} \frac{\sigma_{j}}{\epsilon_{0} D}\left(D-x_{j}\right)-\sum_{x_{i}>x_{j}} \frac{\sigma_{j}}{\epsilon_{0} D} x_{j},
$$

where the first (second) summation accounts for the portion of the space charge field due to the electrons lying above (below) $x_{i}$. Equation (A4) may be rearranged to read as

$$
E\left(x_{i}\right)=-\frac{V_{g}}{D}+\sum_{x_{i}<x_{j}} \frac{\sigma_{j}}{\epsilon_{0}}-\sum_{j} \frac{\sigma_{j}}{\epsilon_{0} D} x_{j},
$$

where the last summation is over all $x_{j}$, i.e., over all space charge. Upon using Eq. (1) of the main text, (A5) becomes

$$
\begin{aligned}
E\left(x_{i}\right) & =-\frac{V_{g}}{D}+\frac{J}{\epsilon_{0}} \sum_{x_{i}<x_{j}} \Delta t_{j}-\frac{J}{\epsilon_{0} D} \sum_{j} \Delta t_{j} x_{j}\left(t, t_{j}\right) \\
& =-\frac{V_{g}}{D}+\frac{J}{\epsilon_{0}} t_{i}-\frac{J}{\epsilon_{0} D} \sum_{j} \Delta t_{j} x_{j}\left(t, t_{j}\right),
\end{aligned}
$$

where we have used the fact that $x_{i}$ is the location of the electron at time $t$ that was released from the cathode at an earlier time $t_{i}$. It is clear from (A6) that $-E\left(x_{i}\right)$, in the continuum limit, is the large parentheses in Eq. (8). Note that Eq. (A6) is exact, no approximation has been used.

For $t>t_{T}$ (but less than $2 t_{T}$ ), the lead electron is returning to the cathode. Let us calculate only the electric field experienced by the lead electron. This calculation is approximate. Let $x_{1}$ be the position of the lead electron (Fig. 5), which is released from the cathode at time $t_{i}=t_{1}$, and $t_{1}=0$ 
by definition. The electric field at $x_{1}$ is still governed by (A4), (A5), and the first part of (A6), which becomes, with $x_{i}=x_{1}$,

$$
E\left(x_{1}\right)=-\frac{V_{g}}{D}-\frac{J}{\epsilon_{0} D} \sum_{j} \Delta t_{j} x_{j}\left(t, t_{j}\right)+\frac{J}{\epsilon_{0}} \sum_{x_{1}<x_{j}} \Delta t_{j} .
$$

The last summation is the total injection time interval between the lead electron and all the subsequent ones whose $x$ coordinates lie above $x_{1}$ at time $t$. Now referring to Fig. 5(a), let $A$ be the lead electron, and $B$ be the last electron, which just arrives at $x_{1}$ at time $t$. The injection time of $A$ is zero, by definition. The injection time of $B$ is $t_{B}$, which we need to calculate. Note that $t_{B}$ is the last summation in Eq. (7).

To facilitate the calculation of $t_{B}$, we now assume that all electrons follow the same trajectories. Specifically, the lead electron has the trajectory, $x\left(t^{\prime}, 0\right)$ as a function of $t^{\prime}$, as shown in Fig. 5(b) and such a trajectory is repeated by all subsequent electrons. We further approximate that this trajectory is symmetrical about $t^{\prime}=t_{T}$, that is, the portion of trajectory between the time interval $\left(0, t_{T}\right)$ is the mirror image of that between $\left(t_{T}, 2 t_{T}\right)$, as shown in Fig. 5(b). Under these symmetry assumptions, it is clear from Fig. 5(b) that it takes the time interval $2 t_{T}-t$ (measured from the time of its injection from the cathode) for an electron to reach the position $x=x_{1}$ for the first time. Now, referring to Fig. 5(a), if $B$ is to reach the point $x=x_{1}$ at time $t$, then its launch time must be $t-\left(2 t_{T}-t\right)$ which is $2\left(t-t_{T}\right)$. Thus $t_{B}=2\left(t-t_{T}\right)$ and this is the approximate value of the last sum in Eq. (A7).

Thus for $t>t_{T}$, Eq. (A7) gives the approximate electric field experienced by a lead electron:

$$
E\left(x_{1}\right)=-\frac{V_{g}}{D}-\frac{J}{\epsilon_{0} D} \sum_{j} \Delta t_{j} x_{j}\left(t, t_{j}\right)+\frac{J}{\epsilon_{0}} \times 2\left(t-t_{T}\right) .
$$

It is easy to see from (A8) that $-E\left(x_{1}\right)$, in the continuum limit, gives the right-hand member of (17) for $\tau>\tau_{T}$.

\section{APPENDIX B: PERTURBATIVE SOLUTIONS}

In this appendix, we record the zeroth-order solutions $\widetilde{V}_{g 0}(\tau), p_{0}(\tau), \xi_{0}(\tau)$ and the first-order solutions $\widetilde{V}_{g 1}(\tau)$ and $\xi_{1}(\tau)$. The first-order solution $p_{1}(\tau)$ is neither needed nor computed.

The solutions for $\widetilde{V}_{g 0}(\tau), p_{0}(\tau)$, and $x_{0}(\tau)$ read as

$$
\begin{aligned}
\widetilde{V}_{g 0}(\tau)= & \widetilde{V}_{0}\left(1-e^{-\tau / r}\right), \\
p_{0}(\tau)= & \widetilde{V}_{0}\left(\tau-\frac{r^{2} e^{-\tau / r}}{\left(1+r^{2}\right)}\left(\frac{2 r}{1+r^{2}}+\tau\right)\right. \\
& \left.+\frac{1}{\left(1+r^{2}\right)^{2}}\left[2 r^{3} \cos (\tau)-\left(1+3 r^{2}\right) \sin (\tau)\right]\right),
\end{aligned}
$$

$\xi_{0}(\tau)=\widetilde{V}_{0}\left(1-\frac{1}{1+r^{2}}\left[r^{2} e^{-\tau / r}-\cos (\tau)-r \sin (\tau)\right]\right)$.

The solutions for $\widetilde{V}_{g 1}(\tau)$ and $x_{1}(\tau)$ read as

$$
\begin{aligned}
\widetilde{V}_{g 1}(\tau)= & r \widetilde{V}_{0}\left\{\frac { e ^ { - \tau / r } } { ( 1 + r ^ { 2 } ) } \left[\frac{r}{1+r^{2}}\left(\frac{r^{3}\left(5+r^{2}\right)}{\left(1+r^{2}\right)}+\tau\left(r^{2}-1\right)\right)\right.\right. \\
& \left.-\frac{\tau^{2}}{2}\right]-\frac{1}{\left(1+r^{2}\right)^{3}}\left[\left(2 r^{4}-3 r^{2}-1\right) \cos (\tau)\right. \\
& \left.\left.-r\left(5 r^{2}+1\right) \sin (\tau)\right]-1\right\}, \\
\xi_{1}(\tau)= & -2[\tau+\sin (\tau)-\pi] h(\tau-\pi)+\widetilde{V}_{0}\left\{\frac{r^{3} e^{-\tau / r}}{\left(1+r^{2}\right)^{2}}\right. \\
& \times\left[\frac{r}{1+r^{2}}\left(\frac{r\left(r^{4}+4 r^{2}-9\right)}{\left(1+r^{2}\right)}-4 \tau\right)-\frac{\tau^{2}}{2}\right]+(\tau-r) \\
& +\frac{\cos (\tau)}{\left(1+r^{2}\right)^{4}}\left[r\left(15 r^{4}+4 r^{2}+1\right)\right. \\
& \left.+\frac{\tau}{2}\left(1+4 r^{2}+r^{4}-2 r^{6}\right)\right]+\frac{\sin (\tau)}{2\left(1+r^{2}\right)^{3}} \\
& \left.\times\left(\frac{1}{1+r^{2}}\left(10 r^{6}-23 r^{4}-12 r^{2}-3\right)+r \tau\left(1+5 r^{2}\right)\right)\right\} .
\end{aligned}
$$

${ }^{1}$ A. W. Hull, Phys. Rev. 18, 31 (1921).

${ }^{2}$ P. J. Christenson and Y. Y. Lau, Phys. Plasmas 1, 3725 (1994); ibid. Erratum: 3, 4293 (1996).

${ }^{3}$ For zero initial velocity and $B>B_{\mathrm{H}}$, the critical current density is NOT achieved under the space charge limited condition of the zero surface electric field. However, the current density computed under the assumption of the zero surface electric field, as done in Ref. 2, still gives a good estimate. See Ref. 4 for more detail. The "critical" current under the zero electric field assumption for the relativistic regime is given by R. V. Lovelace and E. Ott, Phys. Fluids 17, 1263 (1974), while Ron et al. (Ref. 5) did not invoke such an assumption.

${ }^{4}$ P. J. Christenson, Ph.D. thesis, University of Michigan, Ann Arbor, 1996. ${ }^{5}$ A. Ron, A. A. Mondelli, and N. Rostoker, IEEE Trans. Plasma Sci. PS-1, 85 (1973).

${ }^{6}$ L. Brillouin, Phys. Rev. 67, 260 (1945).

${ }^{7}$ P. J. Christenson and Y. Y. Lau, Phys. Rev. Lett. 76, 3324 (1996).

${ }^{8}$ See, e.g., R. C. Davidson, Physics of Nonneutral Plasmas (AddisonWesley, Redwood City, CA, 1990); J. D. Lawson, Physics of Charged Particle Beams (Oxford University Press, Oxford, 1988).

${ }^{9}$ O. W. Richardson, Proc. Cambridge Philos. Soc. 11, 286 (1901); S. Dushman, Phys. Rev. 20, 623 (1923).

${ }^{10}$ PDP1 (Plasma Device Planar 1 Dimensional), Copyright 1990-1993 Regents of the University of California, Plasma Theory and Simulation Group, Berkeley, CA. Available from Software Distribution Office of ILP, 205 Cory Hall, Berkeley, CA 94720; Electronic mail: software@eecs.berkeley.edu. This is a one-dimensional code that includes all three components of velocity.

${ }^{11}$ S. Ramo, Proc. IRE 27, 584 (1939); see also, M. V. Chodorow and C. Susskind, Fundamentals of Microwave Electronics (McGraw-Hill, New York, 1965).

${ }^{12}$ C. K. Birdsall and W. B. Bridges, Electron Dynamics of Diode Regions (Academic, New York, 1966).

${ }^{13}$ Y. Y. Lau, P. J. Christenson, and D. Chernin, Phys. Fluids B 5, 4486 (1993).

${ }^{14}$ A. Palevsky, G. Bekefi, and A. Drobot, J. Appl. Phys. 52, 4938 (1981).

${ }^{15}$ D. Chernin, A. Drobot, G. Hilfer, M. Kess, and S. Riyopoulos, in Digest of IEEE International Electron Devices Meeting (Institute of Electrical and Electronic Engineers, New York, 1991), IEEE Cat. No. 91CH3075-9, p. 593; G. E. Dombrowski, IEEE Trans. Electron Devices ED-35, 2060 (1988); R. MacGregor, C. Chan, J. Ye, and T. Ruden, IEEE Trans. Electron Devices, ED-41, 1456 (1994).

${ }^{16}$ See, e.g., P. G. Drazin, Nonlinear Systems (Cambridge University Press, Cambridge, 1992). 